Laser Chem., 1999, Vol. 19, pp. 1-10

Reprints available directly from the publisher

Photocopying permitted by license only
(C) 1999 OPA (Overseas Publishers Association) N.V. Published by license under the Harwood Academic Publishers imprint, part of The Gordon and Breach Publishing Group.

\title{
INFRARED SPECTRAL HOLE BURNING OF 1:1 HYDROGEN-BONDED COMPLEXES IN SOLUTION*
}

\author{
S. M. ARRIVO ${ }^{\text {a }}$, V. D. KLEIMAN ${ }^{\text {b }}$, W. T. GRUBBS ${ }^{c}$, \\ T. P. DOUGHERTY ${ }^{a}$ and E. J. HEILWEIL ${ }^{d, \dagger}$ \\ a Department of Chemistry and Physics, Beaver College, Glenside PA 19038; \\ Merck and Co., WP 38-3 P.O. Box 4, Westpoint PA 19486, USA; \\ ${ }^{\mathrm{b}}$ Guest scientist from the Dept. of Chemical Engineering, UCLA Los Angeles, \\ CA; Naval Research Laboratory, Washington, DC 20375, USA; \\ ${ }^{\mathrm{c}}$ Department of Chemistry, Stetson University, DeLand FL 32720; \\ ${ }^{\mathrm{d}}$ Room B208 Building 221, Optical Technology Division, National Institute of \\ Standards and Technology, Gaithersburg, MD 20899-0001, USA
}

(Received 21 May 1997)

Transient picosecond infrared (IR) spectra of the $\mathrm{OH}$ and $\mathrm{NH}$-stretch $(v=0-1)$ absorption bands of several hydrogen-bonded complexes have been investigated. Solutions of 1:1 complexes of weak acids (methanol, triethylsilanol and pyrrole, $<0.1 \mathrm{~mol} / \mathrm{dm}^{3}$ ) and bases (acetonitrile, pyridine and tetrahydrofuran, $<2 \mathrm{~mol} / \mathrm{dm}^{3}$ ) in $\mathrm{CCl}_{4}$ at $295 \mathrm{~K}$ were interrogated with IR excitation and broadband probing. Lorentzian-shaped absorption bands are uniformly bleached while those with near-Gaussian bandshapes produce transient spectral holes. These results indicate a base functionality and hydrogen-bond strength dependence for determining the broadening mechanisms of these absorptions.

Keywords: Hydrogen-bond; hole-burning; infrared; broadband; complexes; inhomogeneous

\section{INTRODUCTION}

Picosecond infrared hole burning has previously been investigated for hydrogen-bonded systems including polymer films (polyvinyl-butyral

\footnotetext{
* This paper is dedicated to the memory of Dr. Thomas P. Dougherty - a devoted scientist, colleague, friend and family member who, through his accomplishments and talents, is an inspiration to us all.

${ }^{\dagger}$ Corresponding author.
} 
and polyvinyl-butanal) containing $\mathrm{OH}$-stretch moieties [1], concentrated methanol [2] and ethanol [3] in $\mathrm{CCl}_{4}$ solution at room temperature and in $\mathrm{OH}$-stretching bands of zeolites [4]. For the polymer systems, $a \approx 70 \mathrm{~cm}^{-1}$ FWHM spectral hole burned into the $170 \mathrm{~cm}^{-1}$ wide asymmetric $\mathrm{OH}(v=0-1)$ absorption band had a recovery time of $5 \pm 2 \mathrm{ps}$. Similar results were obtained for the alcohol/ $\mathrm{CCl}_{4}$ solutions. IR spectral holes were attributed to site specific subgroup absorptions or oligomeric species with characteristic vibrational frequencies. Earlier studies performed in this laboratory on hydrogen-bonded $\mathrm{OH}$-stretching bands in micas [5], silica [6], and on silica surfaces [7] revealed frequency-dependent $T_{1}$ vibrational lifetimes and it was concluded that these systems exhibit inhomogeneously broadened absorptions. It was previously shown that the $T_{1}$ lifetime of the NH-stretch mode of pyrrole in a series of hydrogenbonded 1:1 complexes in solution decreases monotonically with increasing base strength (Kamlet-Taft parameter) [8]. This trend also correlates with an increasing frequency red-shift from the monomer absorption frequency [8]. Investigation of the picosecond bleaching dynamics of hydrogen-bonded pyrrole $\mathrm{NH}$-stretch absorption bands via a coarse, narrow frequency probe step method showed that weakly hydrogen-bonded systems produce uniformly bleached absorptions while more strongly bonded systems can produce spectral holes [9]. Weaker hydrogen bonding systems (e.g., pyrrole with ethyl acetate, acetonitrile, acetone, and tetrahydrofuran) produce Lorentzianshaped absorption profiles while stronger H-bonding complex absorptions (e.g., pyrrole with pyridine and triethylamine) are better fit to a Gaussian function. Other hydrogen-bonded systems were also examined for spectral hole burning with IR excitation of the complex $(v=0-1)$ absorption band [10].

We also recently reported transient bleaching dynamics obtained by two-color IR bleaching double-resonance overtone absorption experiments of two similar systems [11]. For triethylsilanol (TES) complexed with acetonitrile $(\mathrm{ACN})$ in $\mathrm{CCl}_{4}$ and TES in neat $\mathrm{ACN}$, the complex $\mathrm{OH}$-stretch $(v=0-1)$ absorption bands and transient bleaching spectra fit a static Lorentzian bandshape. Broadband spectral probing may reveal interesting dynamics that were missed by coarse frequency scanning in these earlier experiments. With the unique capabilities of a transient IR spectrometer for IR excitation and broadband probing 
$\left(15 \mathrm{~cm}^{-1}\right.$ spectral and $1 \mathrm{ps}$ time resolution), we report studies of the bleaching dynamics of $\mathrm{NH}$ and $\mathrm{OH}$-stretch $(v=0-1)$ absorption bands in systems with varying $\mathrm{H}$-bond strengths. By undertaking this study, we gain insight into the microscopic dynamics and structural properties of complexes which produce the static IR absorption bandshapes. In contrast to all previous IR hole-burning experiments, the results reported here were obtained from systems which are ostensively dilute homogeneous room temperature solutions.

\section{EXPERIMENTAL}

The experimental apparatus used to perform these studies was previously described in detail [8]. Three amplified $(20 \mathrm{~Hz})$, tunable, synchronously-pumped (via grating-compressed $\mathrm{CW} \mathrm{Nd}^{+3}$ :YAG SHG) dye lasers were used to generate two independently tunable IR pulses by down-conversion in $\mathrm{LiIO}_{3}$ crystals. Pump pulses were 2 ps FWHM in duration, with $8 \mathrm{~cm}^{-1}$ FWHM bandwidth and $4 \mu \mathrm{J}$ energy at the sample. These pulses were used to excite samples from 3550 to $3350 \mathrm{~cm}^{-1}$. Broadband probe pulses were split into two beams before the sample; both passed through a flowing liquid cell $\left(\mathrm{CaF}_{2}\right.$ windows, $0.82 \mathrm{~mm}$ pathlength) where one was overlapped with the pump pulse (probe) and the other served as a reference. Direct IR probe spectral detection was accomplished with a $256 \times 256$ element mid-IR InSb focal plane array [12]. The signal spectrum was alternately ratioed against the reference spectrum with $(T)$ and without $\left(T_{0}\right)$ the pump beam present to yield signal/reference difference spectra normalized on each laser shot. Kinetic traces were collected by moving an optical delay line in the probe beam path by a computer-controlled translation stage ( $\pm 1 \mu \mathrm{m}$ position accuracy).

Transient absorption difference spectra are presented as the natural logarithm of the transmitted beam divided by the reference beam, $\left[\ln \left(T / T_{0}\right)\right]$. This quantity is directly proportional to the $v=1$ population (via an optical density change) of the probed vibrational mode. Typical absolute errors in $\ln \left(T / T_{0}\right)$ for a single kinetic trace were \pm 0.005 and as low as \pm 0.001 for multiply-averaged data. The instrument response function (IRF) and $t_{d}=0$ (pump-brobe maximum temporal overlap) were determined from pump-probe IR second 
harmonic cross-correlations using a $1 \mathrm{~mm}$ thick $\mathrm{LiIO}_{3}$ crystal. All IR experiments were collected with the pump-probe polarizations at the magic angle ( 54.7 degrees) to eliminate transient contributions from excitation or molecular reorientation. Commercially available acids, bases and spectroscopic grade $\mathrm{CCl}_{4}$ were used as received except acetonitrile which was dried over molecular sieves prior to use. Absorption spectra for each sample in a $1 \mathrm{~mm}$ pathlength IR cell are shown in Figures 1, 2 and 3 and have peak optical densities of $\leq 0.6$.

\section{RESULTS AND DISCUSSION}

The room temperature static FTIR spectrum of a ternary solution of triethylsilanol $\left(\approx 0.1 \mathrm{~mol} / \mathrm{dm}^{3}\right)$, acetonitrile $\left(\approx 1.4 \mathrm{~mol} / \mathrm{dm}^{3}\right)$, and

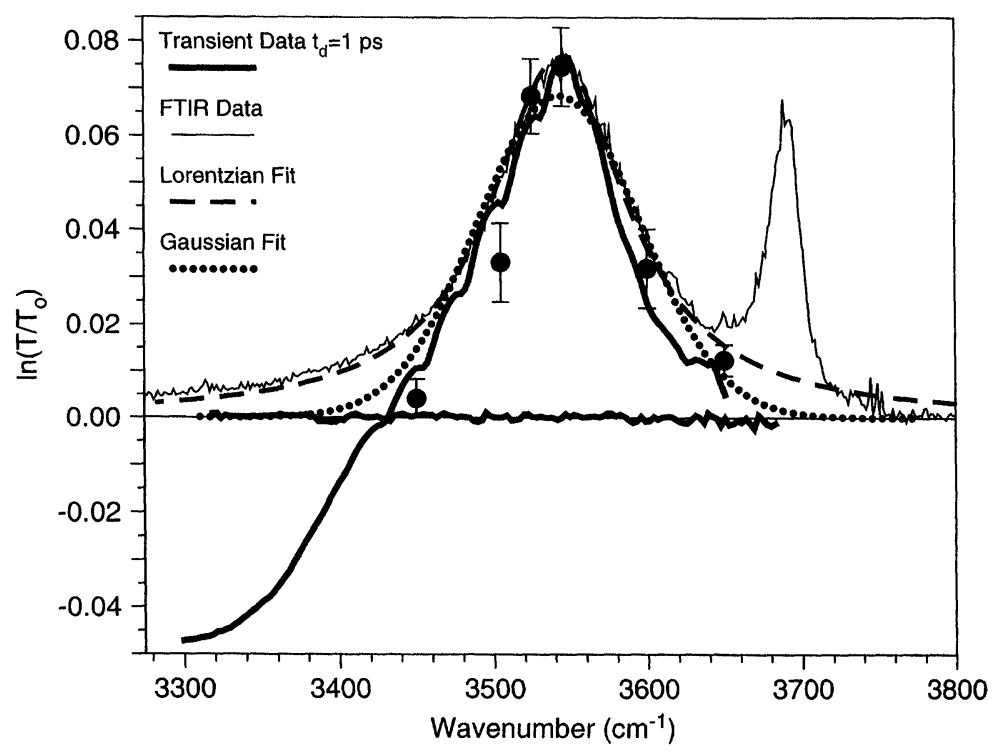

FIGURE 1 Transient IR spectrum (solid line) after IR excitation of the $\mathrm{OH}$-stretch (at $3550 \mathrm{~cm}^{-1}$ ) absorption of dilute 1:1 hydrogen-bonded triethylsilanol-acetonitrile complexes in $\mathrm{CCl}_{4}$ at $295 \mathrm{~K}$. This result and baseline are an average of three data files, 4000 laser shots each. The filled circles are derived from using narrowband probe pulses $\left(10 \mathrm{~cm}^{-1} \mathrm{FWHM}\right)$ with the pump pulse tuned to the absorption center frequency. The narrow line is the static FTIR spectrum of the same room temperature sample (maximum $\mathrm{OD}=0.5$ ). See text for further details of curve fitting procedures. 


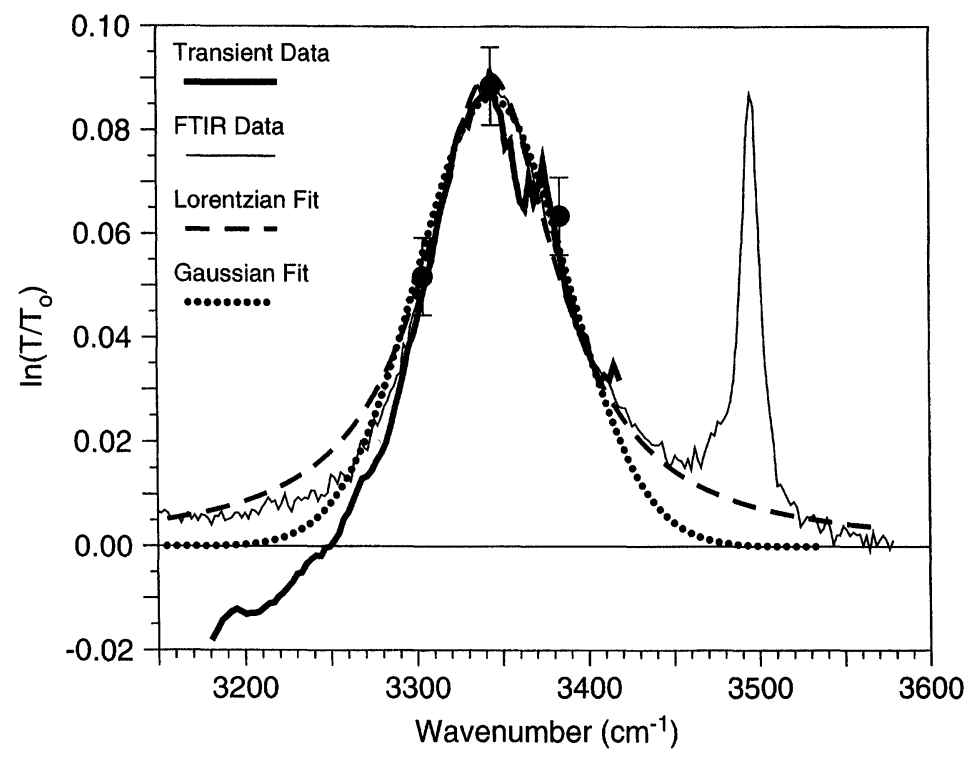

FIGURE 2 Transient IR spectrum (solid line) after IR excitation of the NH-stretching mode absorption (at $3345 \mathrm{~cm}^{-1}$ ) of dilute 1:1 hydrogen-bonded pyrrole-tetrahydrofuran complexes in $\mathrm{CCl}_{4}$ at $295 \mathrm{~K}$. See Figure 1 and text for further details.

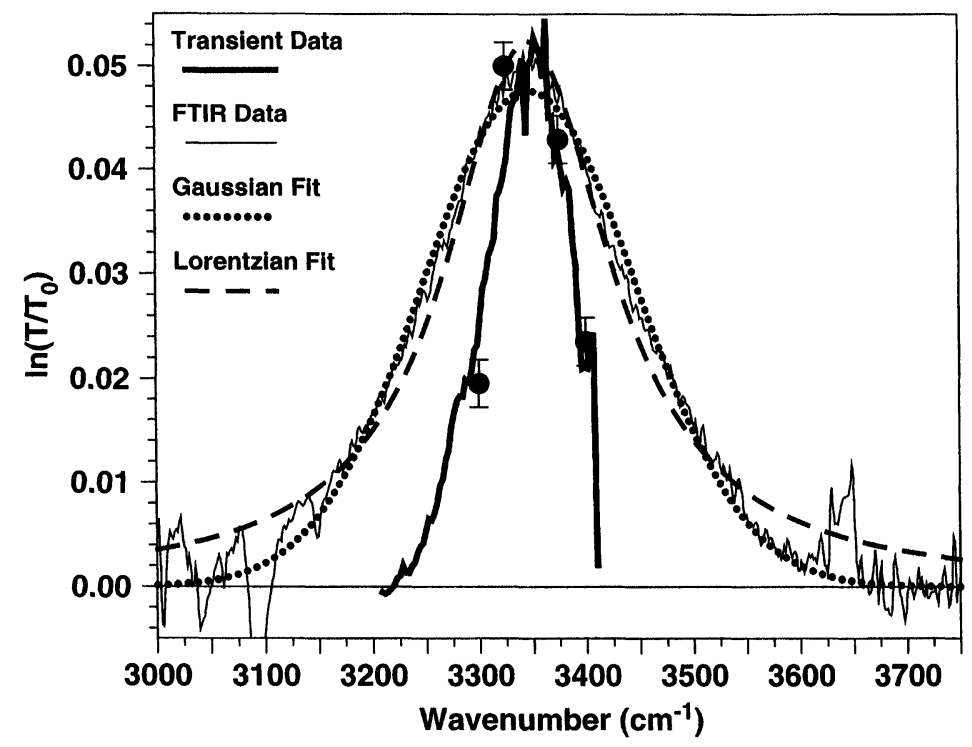

FIGURE 3 Transient IR spectrum (solid line) after IR excitation of the $\mathrm{OH}$-stretching mode absorption (at $3350 \mathrm{~cm}^{-1}$ ) of dilute 1:1 hydrogen-bonded methanol-pyridine complexes in $\mathrm{CCl}_{4}$ at $295 \mathrm{~K}$. See Figure 1 and text for further details. 
carbon tetrachloride is shown as the narrow line in Figure 1. The transient bleaching spectrum of this system was collected by IRexcitation of the $\mathrm{OH}$-stretching $(v=0-1)$ mode absorption of the hydrogen-bonded TES:ACN complex at $3550 \mathrm{~cm}^{-1}\left(112 \mathrm{~cm}^{-1}\right.$ FWHM). Data at $1.0 \mathrm{ps}$ delay were smoothed with a three point running average algorithm and shown in Figure 1 (solid line). The positive feature arises from bleaching of the $\mathrm{OH}(v=0-1)$ vibrational transition by the pump pulse, while the negative feature arises from $\mathrm{OH}(v=1-2)$ absorption of the excited complex. The static FTIR absorption spectrum is scaled to match the bleach intensity at the peak maximum. Note the excellent agreement between the transient bandshape and static FTIR data. These results in conjuction with transient decay data (giving nominally the same 25 ps bleach recovery time at several excitation frequencies) confirm our conclusions from previous narrowband investigations on two issues. First, the band appears to be homogeneously broadened (the entire band bleaches uniformly within $2 \mathrm{ps}$ ). Secondly, the bleach and absorption band intensities uniformly return to the baseline with the previously determined $25 \pm 5 \mathrm{ps}$ lifetime [11]. Also shown in Figure 1 is a Lorentzian functional fit to the complex $\mathrm{OH}(v=0-1)$ FTIR absorption band (dashed line). The Lorentzian profile and the uniform bleaching dynamics suggests the absorption band is homogeneously broadened within the instrumental timescale of 1 to $2 \mathrm{ps}$.

The transient IR bleaching spectrum of the NH-stretch $(v=0-1)$ absorption band of pyrrole $\left(0.05 \mathrm{~mol} / \mathrm{dm}^{3}\right)$ complexed with tetrahydrofuran (THF) $\left(0.7 \mathrm{~mol} / \mathrm{dm}^{3}\right)$ in $\mathrm{CCl}_{4}$ is shown in Figure 2 (solid line). The narrow line in this figure represents the static FTIR spectrum of this hydrogen-bonded complex. The complex absorption maxima at $3350 \mathrm{~cm}^{-1}$ is red-shifted from the pyrrole monomer $\mathrm{NH}$ stretch absorption by approximately $145 \mathrm{~cm}^{-1}$, indicating a reasonably strong hydrogen-bonding interaction [8]. The dashed line is a Lorentzian fit $\left(103 \mathrm{~cm}^{-1}\right.$ FWHM) to the NH-stretch FTIR absorption profile. The Lorentzian bandshape suggests that the absorption is homogeneously broadened. The peak amplitude of the FTIR spectrum is normalized to match the peak of the transient bleach, and it is clear that the entire absorption profile is bleached after NH-stretch ( $v=0-$ 1) excitation at $3350 \mathrm{~cm}^{-1}$. Note the close agreement between the $(v=0-1)$ FTIR absorption spectrum and the transient bleaching 
spectrum. Bleaching of the entire absorption band, uniformity of recovery time [8], and the best fit Lorentzian profile strongly suggests that the absorption band for this system is also homogeneously broadened within the 1-2ps timescale.

Previous narrowband pump-probe bleaching experiments on pyrrole:THF complexes with data points at the center frequency and half maxima of the absorption band also indicated a homogeneous absorption and yielded an approximately 4 ps bleaching recovery time [8]. These data points are included in Figure 2 as filled dots. In the lower frequency region, the onset of NH-stretch $(v=1-2)$ excited state absorption is apparent. We also reproduced the $T_{1}$ recovery time of the complex $\mathrm{NH}(v=0-1)$ absorption band $(4 \pm 2 \mathrm{ps})$ with the broadband probe methodology [8]. This measurement was performed by restricting the spectral content of the probed region to the bleaching band and performing kinetic measurements of the total spectrally integrated band intensity as a function of time delay.

To further understand the H-bonding absorption characteristics, we also investigated bleaching dynamics of the more strongly hydrogenbonded system methanol:pyridine: $\mathrm{CCl}_{4}$ with the same IR pump broadband IR probe method. Shown in Figure 3 is the FTIR absorption spectrum of a dilute solution of methanol $\left(0.1 \mathrm{~mol} / \mathrm{dm}^{3}\right)$ and pyridine $\left(0.6 \mathrm{~mol} / \mathrm{dm}^{3}\right)$ in room temperature $\mathrm{CCl}_{4}(\mathrm{MeOH}$ :pyridine complex) and spectral fits using Lorentzian and Gaussian functions. The measured FWHM of the absorption band is $220 \mathrm{~cm}^{-1}$ and the peak frequency is red-shifted from the dilute methanol $\mathrm{OH}$-stretch $(v=0-1)$ absorption by approximately $295 \mathrm{~cm}^{-1}$. The Gaussian fit more closely resembles the FTIR absorption spectrum for the hydrogen-bonded $\mathrm{OH}$-stretch [13]. The near-Gaussian profile suggests the vibration is perturbed by an inhomogeneous spectral broadening mechanism. This condition could produce a transient spectral hole when a narrowband IR excitation pulse is used to excite the complex absorption. The possibility of inhomogeneous broadening and spectral hole burning of this hydrogen-bonded complex $\mathrm{OH}(v=0-1)$ absorption band was investigated with a $1 \mathrm{ps}$, ca. $12 \mathrm{~cm}^{-1}$ FWHM IR pump pulse. The solid line in Figure 3 is the resultant bleaching spectrum at $t_{d}=1.0 \mathrm{ps}$ after IR excitation $\left(3350 \mathrm{~cm}^{-1}\right)$ of the $\mathrm{MeOH}$ :pyridine sample. The vibrational lifetime of the $\mathrm{MeOH}$ :pyridine complex was previously measured to be $1.5 \pm 1 \mathrm{ps}$ [14]. This $T_{1}$ 
lifetime is likely to be shorter than the rotational time constant of the complex, and prevents observation of spectral diffusion of the transient hole since the population recovery time is very near our instrumental time resolution. The observed transient bleaching (spectral hole) is $80 \pm 10 \mathrm{~cm}^{-1} \mathrm{FWHM}$, about 2.8 times narrower than the static FTIR spectrum shown in Figure 3. Observation of similar spectral hole burning from $N$-methylacetamide dimer and complexes will be reported in a subsequent publication [15].

We believe a distribution of long-lived ( $>2 \mathrm{ps}$ ) hydrogen-bonded complex geometries (giving rise to different absorption frequencies) are responsible for the observed transient spectral hole in the methanol:pyridine system. Further experiments performed as a function of temperature and time delay may help solidify this argument. In order to investigate the possibility of inhomogeneous broadening caused by a distribution of conformational states, obtaining static FTIR spectra at lower temperatures may "freeze-out" high energy conformer populations and alter the absorption spectral width or shape. It would be interesting to see if the FTIR spectrum narrows at attainable lower temperatures to the same extent as observed for the spectral hole burnt in this system. Further detailed investigation of the hole-filling dynamics may also reveal geometrical interconversion rates and energies.

It is possible that $\mathrm{ACN}$, being the lightest and weakest base $\left(K_{\mathrm{eq}} \approx 1\right.$ with TES), is retained near the proton-donating TES acid by the heavy $\mathrm{CCl}_{4}$ solvent and keeps the distribution of $\mathrm{H}$-bonding geometries to a minimum. The distributions of geometries in the weaker $\mathrm{H}$-bonded systems may be less perturbed and the center frequency and width of the $\mathrm{H}$-bond absorption arises from rapid dephasing or other mechanisms. Sub-picosecond hindered rotation of the methyl group of ACN may directly contribute to the dephasing bandwidth by indirect coupling to the $\mathrm{OH}$-stretch through the low frequency $\left(<200 \mathrm{~cm}^{-1}\right)$ hydrogen-bond stretch. The same arguments may apply to the only slightly stronger THF complex with pyrrole. On the other hand, spatially directed nitrogen electron donation of pyridine to the methanol hydroxyl proton may yield preferred conformational orientations with distinct frequencies so that IR pump pulses excite a fraction of the conformer population. Further experiments with heavy nitriles and those with larger electron-donating capability may 
address these notions and clarify the origin of the width of $\mathrm{OH}$ and $\mathrm{NH}$-stretches in hydrogen-bonded complexes.

\section{CONCLUSIONS}

We observe a correlation between static FTIR $v=0-1$ absorption band profiles for $\mathrm{NH}$ and $\mathrm{OH}$-stretches, $\mathrm{H}$-bond strength, and transient IR spectral hole burning in hydrogen-bonded complexes. More weakly bound complexes (H-donors with acetonitrile, THF) exhibit homogeneous, Lorentzian bandshape bleaching and population recovery dynamics longer than the 1 to 2 ps instrumental time resolution. Methanol complexed to the strong base pyridine, exhibits a near-Gaussian absorption profile and produces an IR spectral hole. Femtosecond IR spectra or photon echo experiments may help elucidate whether collisional dephasing or other mechanisms are responsible for hole burning in these solution-phase systems.

\section{References}

[1] Graener, H. and Laubereau, A. (1991). J. Phys. Chem., 95, 3448; Graener, H. (1991). J. Phys. Chem., 95, 3450; Graener, H., Losch, T. and Laubereau, A. (1990). J. Chem. Phys., 93, 5365.

[2] Bonn, M., Brugmans, M. J. P., Kleyn, A. W., van Santen, R. A. and Bakker, H. J. (1996). J. Chem. Phys., 105, 3431; Bonn, M., Bakker, H. J., Kleyn, A. W. and van Santen, R. A. (1996). J. Phys. Chem., 100, 15301.

[3] Graener, H., Ye, T. Q. and Laubereau, A. (1989). J. Chem. Phys., 91, 1043; ibid., (1989). J. chem. Phys., 90, 3413; Laenen, R. and Rauscher, C. (1997). J. Chem. Phys., 106, xxx; Laenen, R., Rauscher, C. and Laubereau, A. (1997). J. Phys. Chem., A101, xxx.

[4] Bonn, M., Brugmans, M. J. P., Bakker, H. J., Kleyn, A. W. and van Santen, R. A. (1996). Stud. Surf. Sci. Cat., 97, 567; Fujino, T., Fuauki, M., Kashitani, M., Onada, K., Kubota, J., Kondo, J. N., Wada, A., Domen, K., Hirose, C., Wakabayashi, F., Ishia, M., Goto, F. and Kano, S. S. (1996). J. Chem. Phys., 105, 279.

[5] Heilweil, E. (1986). Chem. Phys. Letters, Vol. 129, 48.

[6] Heilweil, E. J., Casassa, M. P., Cavanagh, R. R. and Stephenson, J. C. (1985). Chem. Phys. Lett., Vol. 117, 185.

[7] Heilweil, E. J., Casassa, M. P., Cavanagh, R. R. and Stephenson, J. C. (1989). Ann. Rev. Phys. Chemistry, Vol. 40, 143.

[8] Grubbs, W. T., Dougherty, T. P. and Heilweil, E. J. (1995). J. Phys. Chem., 99, 10716.

[9] Grubbs, W. T., Arrivo, S. M. and Heilweil, E. J., unpublished results.

[10] Experiments on MeOH:THF were also conducted. The $\mathrm{OH}(v=0-1)$ absorption profile is fit equally well by either a Lorentzian or Gaussian function. Either case is reasonable since methanol has a comparable basicity to $\mathrm{THF}^{8}$ and we have shown 
that THF forms homogeneously broadened complexes with triethylsilanol and pyrrole. Transient kinetics and spectra indicate homogeneous broadening to be the dominant mechanism since most of the $\mathrm{OH}(v=0-1)$ absorption band bleaches after IR excitation.

[11] Arrivo, S. M. and Heilweil, E. J. (1996). J. Phys. Chem., 100, 11975.

[12] Arrivo, S. M., Kleiman, V. D., Dougherty, T. P. and Heilweil, E. J. (1997). Optics Letters, 22, 1488.

[13] $\sum\left(X^{2}\right)=1.43$ for the Gaussian fit and $\sum\left(X^{2}\right)=2.1$ for the Lorentzian fit.

[14] Grubbs, W. T., Arrivo, S. M. and Heilweil, E. J., unpublished results.

[15] $N$-methylacetamide complexed to THF (weakly hydrogen-bonded) and NMA dimer/oligomer (more strongly $\mathrm{H}$-bonded) were also investigated. For monomeric NMA in $\mathrm{CCl}_{4}$ or $\mathrm{CHCl}_{3}$, the $T_{1}$ vibrational lifetime of the $\mathrm{NH}(v=1)$ stretching population is $13 \pm 2 \mathrm{ps}$. The NMA/THF complex NH $(v=0-1)$ absorption profile is best fit by a Lorentzian and the band bleaches uniformly with IR pumping at the complex absorption peak $\left(3320 \mathrm{~cm}^{-1}\right)$. A $0.08 \mathrm{~mol} / \mathrm{dm}^{3}$ solution of NMA also has an intense hydrogen-bonded NH-stretch absorption at $3320 \mathrm{~cm}^{-1}$ from selfassociated NMA. This absorption profile has Gaussian character and a spectral hole was produced after IR-pumping at the peak of this absorption band. 